\title{
Cephalexin Degradation Initiated by OH Radicals: Theoretical Prediction of the Mechanisms and the Toxicity of Byproducts
}

R. Masmoudi

Universite de Batna: Universite Hadj Lakhdar Batna

S. Khettaf

Universite Hadj Lakhdar Batna

\section{A. Soltani}

Universite Hadj Lakhdar Batna

A. Dibi

Universite de Batna: Universite Hadj Lakhdar Batna

L. Messaadia

Universite de Jijel

Messaoud BENAMIRA ( $\sim$ benamira18@yahoo.fr)

Universite de Jijel https://orcid.org/0000-0002-2460-9457

\section{Research Article}

Keywords: Quantum chemical calculations, DFT, Advanced oxidation processes, Cephalexin, Hydroxyl radical, Wastewater treatment

Posted Date: December 30th, 2021

DOI: https://doi.org/10.21203/rs.3.rs-1169649/v1

License: (9) This work is licensed under a Creative Commons Attribution 4.0 International License.

Read Full License 


\title{
Cephalexin degradation initiated by $\mathrm{OH}$ radicals: Theoretical prediction of the mechanisms and the toxicity of byproducts
}

\author{
R. Masmoudi ${ }^{1}$, S. Khettaf $^{1}$, A. Soltani ${ }^{1}$, A. Dibi ${ }^{1}$ L. Messaadia $^{2}$ [0000-0003-2542-0098] \\ , M. Benamira ${ }^{3, *}[0000-0002-2460-9457]$ \\ ${ }^{1}$ Laboratory of Chemistry and Environmental Chemistry LCEE, Department of Chemistry, \\ Faculty of Material Sciences, University of Batna 1, Batna, 05000, Algeria \\ ${ }^{2}$ Laboratory of Applied Energy and Materials (LEAM), University of Jijel, BP. 98, Ouled Aissa \\ 18000, Jijel, Algeria \\ ${ }^{3}$ Laboratory of Interaction Materials and Environment (LIME), University of Jijel, BP. 98, \\ Ouled Aissa 18000, Jijel, Algeria \\ *Corresponding author: redhachem@yahoo.fr,m_benamira@univ-jijel.dz.
}

\begin{abstract}
In this work, density functional theory is used to study the local reactivity of cephalexin (CLX) to radical attack and explain the mechanism of the reaction between CLX and hydroxyl radical attack leading to degradation byproducts. The reaction between $\bullet \mathrm{OH}$ and CLX is supposed to lead to either an addition of a hydroxyl radical or an abstraction of a hydrogen. The results showed that the affinity of cephalexin for addition reactions increases as it passes from the gas to the aqueous phase and decreases as it passes from the neutral to the ionized form. Thermodynamic data confirmed that $\mathrm{OH}$ addition radicals $\left(R_{\text {add }}\right)$ are thermodynamically favored over $\mathrm{H}$ abstraction radicals $\left(R_{\text {abs }}\right)$. The ecotoxicity assessments of CLX and its byproducts are estimated from the acute toxicities toward green algae, Daphnia and fish. The formation of byproducts is safe for aquatic organisms, and only one byproduct is harmful to Daphnia.
\end{abstract}

Keywords: Quantum chemical calculations; DFT; Advanced oxidation processes; Cephalexin; Hydroxyl radical; Wastewater treatment.

\section{Introduction}

Growing concentrations of antibiotics and their metabolites in aqueous environments have caused great concerns to human health and different ecological systems. Cephalexin, $(6 R, 7 R)-$ 7-[[(2R)-2-amino-2-phenylacetyl]amino]-3-methyl-8-oxo-5-thia-1 azabicyclo[4.2.0]oct-2ene-2-carboxylic acid (CLX), is a first generation cephalosporin used in human and veterinary medicine in the treatment of bacterial infections [1]. Cephalexin has a biotransformation rate 
of only $10 \%$, and the remaining $90 \%$ is excreted unchanged in the urine [2]. Cephalexin is frequently detected in the effluents of wastewater treatment plants (WWTPs) and exhibits very low biodegradability [3]. Consequently, the World Health Organization (WHO) warned about the presence of pharmaceuticals in drinking water and the potential implications on human health and the environment and classified cephalosporin antibiotics as emergent environmental contaminants [4]. WWTPs use advanced oxidation processes (AOPs) to remove contaminants from wastewater. This technique is based on the in situ generation of reactive oxygen species (ROS) and hydroxyl radicals $(\bullet \mathrm{OH})$ through chemical processes that employ ozone and hydrogen peroxide combined with irradiation of UV light, electromagnetic radiation, and ultrasound [5-7]. The advantage of $\bullet \mathrm{OH}$ is that it is highly reactive and nonselective with a large range of organic compounds [8]. There are numerous methods for the determination of local reactivity descriptors. The study of He et al. [2] located the reactive sites of cephalexin on the basis of frontier electron densities FEDHомо and FED Lumo for each site. The research established by Cao et al. [9] compared the different methods used for local reactivity determination, and these methods differed in their accuracy. The most accurate approach is the one proposed by Yang and Mortier [10] using the atom condensed Fukui function. The byproduct structures of CLX hydroxyl-mediated degradation have been reported by some authors [11-15], while the thermodynamic data and a mechanism of the degradation process are still uncovered. Previous studies indicate that the most common degradation pathways include hydroxylation, decarboxylation, demethylation and dealkylation. Another study claimed that the majority of oxidation intermediates result from OH attack on the CLX cephalosporin ring, where the intermediates undergo opening of the active site of the molecule responsible for the antibacterial activity. Another intermediate formation pathway involves the electrophilic addition of $\mathrm{OH}$ to the aromatic moiety [16]. Terminal degradation products such as lactic, acetic and propinoic acids are rapidly further oxidized (mineralization processes). However, fumaric, oxalic and oxamic acids are hard to further oxidize. More recently, there has been a growing interest in predicting the photodegradation mechanism, kinetics, and ecotoxicological assessment of water emerging contaminants (EOCs) with the assistance of theoretical calculations. Several experimental studies have been conducted on cephalexin hydroxyl-mediated degradation, sonochemical processes [17], electrochemical advanced oxidation processes [16], photo-Fenton processes [18] and photocatalytic nanowire oxidation processes [2], but little is known about its degradation mechanism. Gathering information on the degradation mechanisms and pathways of cephalexin and the ecotoxicological impact of its byproducts has become an issue of great concern for the treatment of CLX and other 
pharmaceutical pollutants.

\section{Materials and methods}

\subsection{Quantum chemical calculations}

All calculations were performed with the Gaussian 16 program package. Geometry optimization was conducted using the DFT/B3LYP/6-31+G(d) basis set [19]. Stationary points were characterized by frequency analysis where the free energy and thermal enthalpy were obtained at $298 \mathrm{~K}$. Polarizable continuum model (CPCM) was used to study the effect of the solvent environment, i.e., water $(\varepsilon=78.36)$ [20]. The pKa of cephalexin was calculated in this work using the ChemAxon Marvin Sketch software (ChemAxon Kft, Budapest, Hungary). Its value indicated in the work of some authors was 2.56 for the carboxylic function and 6.88 for the primary amine function [21]. Since degradation takes place in a medium at $\mathrm{pH}=7$ (for water), the degradation of CLX was modeled into neutral and anionic forms in both gas and aqueous phases. From the values of the charges of neutral cephalexin $q(N)$, the cation and the corresponding anion $q(N-1), q(N+1)$, respectively, the Fukui indices for electrophilic, nucleophilic and radical attacks, i.e., $f_{k}^{+}, f_{k}^{-}$and $f_{k}^{0}$, respectively, are calculated as follows [22]:

$$
\begin{aligned}
& f_{k}^{+}=q(N+1)-q(N) \text { for nucleophilic attack } \\
& f_{k}^{-}=q(N)-q(N-1) \text { for electrophilic attack } \\
& f_{k}^{0}=\frac{1}{2}[q(N+1)-q(N-1)] \text { for radical attack }
\end{aligned}
$$

Eq. (5) and Eq. (7) were used to calculate the stability of the generated radicals of $\mathrm{OH}$ addition and $\mathrm{H}$ abstraction, respectively [23,24]:

$$
\begin{aligned}
& \mathrm{CLX}+\bullet \mathrm{OH} \rightarrow R_{a d d} \bullet \\
& \Delta G_{r x n}=G\left(R_{a d d \bullet}\right)-G(\bullet \mathrm{OH})-G(C L X) \\
& \mathrm{CLX}+\bullet \mathrm{OH} \rightarrow R_{\mathrm{abs}} \bullet+\mathrm{H}_{2} \mathrm{O} \\
& \Delta G_{r x n}=G\left(H_{2} \mathrm{O}\right)+G\left(R_{a b s}\right)-G(\bullet \mathrm{OH})-G(C L X)
\end{aligned}
$$


In addition to the above measures, the standard radical stabilization energies $\left(\mathrm{RSE}=\Delta H_{298}^{0}\right)$ of the produced radicals were calculated at $298 \mathrm{~K}$ using Eq. (9), where $\mathrm{R} \bullet$ is the concerned radical.

$$
\begin{gathered}
\mathrm{CH}_{4}+\mathrm{R} \bullet \rightarrow \mathrm{CH}_{3} \bullet+\mathrm{R}-\mathrm{H} \\
\Delta H_{298}^{0}=\Delta_{f} H_{298}^{0}\left(\mathrm{CH}_{3} \bullet\right)+\Delta_{f} H_{298}^{0}(\mathrm{R}-\mathrm{H})-\Delta_{f} H_{298}^{0}\left(C_{4}\right)-\Delta_{f} H_{298}^{0}(\mathrm{R} \bullet)
\end{gathered}
$$

$\Delta \mathrm{H}$ is the enthalpy change of the reaction. In the case where it corresponds to a positive value, $\mathrm{R} \bullet$ is expected to be more stable than $\mathrm{CH}_{3} \bullet$; otherwise, $\mathrm{R} \bullet$ is expected to be less stable than $\mathrm{CH}_{3} \cdot$.

The Gibbs free energies of fragmentation help to identify the most suitable fragmentation pathways of CLX/CLX', which are calculated using Eq. (10):

$$
\Delta G_{r x n}=(G(\text { fragment } 1)+G(\text { fragment } 2))-G(C L X)
$$

\subsection{Ecotoxicity assessment}

The ecotoxicological impact of cephalexin and its degradation byproducts were estimated for three tropical levels (fish, green algae and Daphnia) in terms of both efficient and lethal concentrations. Ecological Structure Activity Relationship software (ECOSAR) was used in this study [25]. ECOSAR Class Program is a computerized predictive system used to predict the aquatic toxicity. The program estimates a chemical's acute toxicity and chronic toxicity to aquatic organisms, such as fish, aquatic invertebrates, and aquatic plants, by using computerized Structure Activity Relationships (SARs). EC 50 refers to the pollutant level of the tested compound that could inhibit 50\% growth of green algae after $96 \mathrm{~h}$ of exposure time. $\mathrm{LC}_{50}$ represents the concentration of the tested pollutant for $50 \%$ death of fish and Daphnia after 96 and $48 \mathrm{~h}$ of contact times, respectively.

\section{Results and discussion}

\subsection{Initial reaction of $C L X$ with $\bullet \mathrm{OH}$}

In the aqueous phase, cephalexin can exist in six forms depending on the $\mathrm{pH}$ of the solution (Fig. 1). The ChemAxon Marvin Sketch software was used to calculate the pKa and to predict which structure of acid or base will dominate in the $\mathrm{pH}$ range of $0-14$. In this diagram of the 
species repartition presented in terms of molar fractions (\%), six forms were observed. In the first region ( $\mathrm{pH}$ range of $0-4$ ), the predominant species is $\mathrm{C}$, with molar fractions of $99.91 \%$. The first deprotonation takes place at the $\mathrm{OH}$ of carboxylic acid, with a relative $\mathrm{pKa}$ value of 3.26 (Fig. 2). The second region is described by the $\mathrm{B}$ species from $\mathrm{pH} 7$ to 12 with molar fractions of $99.09 \%$. In this case, deprotonation took place at the primary amine $\mathrm{NH}_{2}$ functional group $(\mathrm{pKa}=7.23)$, and these results are in good agreement with the literature $[26,27]$. In the third region, from $\mathrm{pH}=4$ to 7 , the most important species is $\mathrm{D}$ in a zwitterion form with a molar fraction of $97.95 \%$. The other species (E, F and $\mathrm{G})$ existed in the $\mathrm{pH}$ region of 10-14 with molar fractions of 53.43, 12.40 and $94.97 \%$, respectively.

Cephalexin has two functions mainly responsible for the acid-base behavior in the molecule, a carboxylic acid function and primary amine, and the degradation of cephalexin takes place in a medium at $\mathrm{pH}=7$ (for water). This allows us to model the molecule in both neutral and anionic forms. The solvent intervenes in the geometric parameters by influencing, mainly, the bonds of the aliphatic chain and, by a less remarkable effect, the bonds of the cephem ring, while the lengths of the bonds of benzene are not practically affected. The most stable conformers of the neutral and anionic forms are depicted in Fig. 3.

Advanced oxidation products (AOPs) produce powerful nonselective oxidant agents, mainly -OH radicals, which interact with contaminant molecules to initiate the degradation process [7]. Information on the behavior of cephalexin in the presence of $\bullet \mathrm{OH}$ radicals is first required to determine the most likely sites in view of radical attack. The reactivity of cephalexin toward - $\mathrm{OH}$ is supposed to be either by hydrogen abstraction or hydroxyl addition. The concerned sites on cephalexin molecules are either hydrogen atoms, in which the outcome of their interaction with hydroxyl radicals is an abstraction reaction, or unsaturated carbon atoms, in which the result of their interaction with hydroxyl radicals is an addition reaction [28]. The reactivity of the candidate sites is studied through atom condensed Fukui index analysis. The Fukui indices for neutral and ionized cephalexin in gas and aqueous phases are calculated with equations 1, 2 and 3; the results are summarized in Tables 1 to 4, respectively. The Fukui indices were determined to predict the initial attack position during the photocatalytic degradation by reactive oxygen species (ROS). According to the Table1 (in gas phase), the most positive atoms with higher values of $f_{k}^{+}$function is localized at C25 (0.46), C31 (0.04) and C40 (0.01). However, in aqueous phases (Table 2), C14 (0.07), H24 (0.17) and $\mathrm{H} 21(0.12)$ atoms showed the more positive values of $f_{k}^{+}$function (atom abstraction by $\bullet \mathrm{OH}$ ). Consequently, the addition of $\bullet \mathrm{OH}$ maybe took place on these atoms (Nucleophilic 
attack). Nevertheless, the most negative part of $f_{k}^{-}$function in gas phase is localized in C25 (-0.14), C26 (-0.31) and C40 (-0.30). Whereas C14 (-0.12), C25 (-0.39), C26 (-0.29) and C40 $(-0.15)$ are regarded as the most favorable sites for electrophilic attack in aqueous phases. In the case of ionized cephalexin in gas phases (Table 3), the atoms with more positive values of $f_{k}^{+}$function are localized in C26 (0.13), C39 (0.03). However, C25 (0.23) and C26 (0.10) atoms in aqueous phases (Table 4) showed the most positive values and the best negative part of $f_{k}^{-}$function are localized in C26 (-0.68) and C31 (-0.10).

The reaction sites extracted from the analysis of atom condensed Fukui indices are circled (in red) and labeled, as shown in Figs. 4 and 5. To facilitate the recognition of the reaction site in future studies, abstraction sites are assigned and labeled with numbers (from 1 to 11), and additional sites are labeled with letters (from $a$ to $e$ ), as shown in Figs. 6 and 7.

The free energies, enthalpies and radical stabilization energies of the abstraction and addition radicals are calculated with equations 7, 9 and 10, and the results are displayed in Tables 5-7, respectively. The most stable abstraction radical corresponds to $\mathrm{R}_{\mathrm{abs}} 5$ (-H38) with the highest stabilization energy (-0.08 kcal/mol). The $\mathrm{R}_{\mathrm{abs}} 3$ (-H9) and $\mathrm{R}_{\mathrm{abs}} 2$ (-H8/H10) radicals have almost the same stability, although the generation of the $\mathrm{R}_{\mathrm{abs}} 3$ (-H9) radical is more energetically favorable (para position) than that of $\mathrm{H} 8$ and $\mathrm{H} 10$ (meta position). The abstraction of an $\mathrm{H}$ proton from positions $10(-\mathrm{H} 34)$ and $4(-\mathrm{H} 13)$ is found to be more favorable than the abstraction of an $\mathrm{H}$ proton from the benzene ring; this is explained on the basis of the stabilization generated by the conjugation of the carboxyl radical and the benzyl radical in the second and third cases. The generation of these three radicals, $R_{a b s} 5, R_{a b s} 3$, and $R_{a b s} 2$, is exergonic and thermodynamically favorable compared to other abstraction reactions.

The abstraction radical $\mathrm{R}_{\mathrm{abs}} 9(-\mathrm{H} 28 / 29 / 30)$ is the most stable radical after the three preceding radicals; its formation is accompanied by energy absorption (endergonic), and its stability is also explained by conjugation with the double bond (allylic radical).

The $\mathrm{R}_{\mathrm{abs}} 11$ (-H20), Rabs7 (-H21) and $\mathrm{R}_{\mathrm{abs}} 8$ (-H23/H24) radicals are the least stable. Their formations are the most endergonic, and their low stability is explained by the absence of stabilizing effects (conjugation and hyperconjugation) next to the radical.

In general, in the gas phase, abstraction radicals are more stable than those produced in the aqueous phase. The most stable addition radical is that corresponding to $R_{a d d}{ }^{e}(C 31)$ with a 
stabilization energy of $0.02 \mathrm{kcal} / \mathrm{mol}$. This radical seems to be more unstable by moving from the neutral form $C L X$ to the anionic form $C L X^{-}(-0.94 \mathrm{kcal} / \mathrm{mol})$.

The $\mathrm{R}_{\mathrm{add}}{ }^{\mathrm{a}}(\mathrm{C} 25)$ radical is the least stable. Its formation is the most endergonic $(0.08 \mathrm{kcal} / \mathrm{mol})$, and its low stability is explained by the fact that the center of the radical is adjacent to the carboxyl group which exerts an inductive attracting effect on it (destabilizing effect). On the other hand, the addition of the $\mathrm{OH}$ radical to the $\mathrm{C} 25$ carbon $\left(\mathrm{R}_{\mathrm{add}}{ }^{\mathrm{a}}(\mathrm{C} 25)\right)$ generates a more stable tertiary radical (because of the inductive donor effect of the three groups that surround it).

The addition of the $\mathrm{OH}$ radical to $\mathrm{C} 14\left(\mathrm{R}_{\mathrm{add}}{ }^{\mathrm{c}}(\mathrm{C} 14)\right)$ and $\mathrm{C} 40\left(\mathrm{R}_{\mathrm{add}}{ }^{\mathrm{d}}(\mathrm{C} 40)\right)$ ends with the breaking of the amide bond (hydrolysis reaction) which is cyclic in the first case and aliphatic in the second case [16,29]. The resistance of the cyclic amide to the 4-chain link is weaker than that of the aliphatic amide, resulting in a high tendency to react, i.e., $-27.73 \mathrm{kcal} / \mathrm{mol}$ compared to $9.85 \mathrm{kcal} / \mathrm{mol}$.

\subsection{Fragmentation $C L X / C L X$}

The fragmentation of CLX/CLX ${ }^{-}$is modeled to predict the possible fates of the generated radical intermediates and to predict the most likely degradation pathways. All fragmentation pathways are shown in Fig. 8. The reaction energies of all optimized fragments are calculated using Eq. (10) and presented in Table 8 in ascending order. Cleavage of the benzylamine moiety (entry 1) appears to be easier than the other fragmentation pathways. In the gas phase, deamination (entry 2) and demethylation (entry 3) have the same difficulty. Moreover, the losses of $(\mathrm{OH})$ (entry 8) in neutral form and $\left(\mathrm{O}^{-}\right)$in anionic form are more endergonic than those of the others. The decarboxylation is less endergonic for $\mathrm{CLX}^{-}(71.84(64.5) \mathrm{kcal} / \mathrm{mol})$ than for CLX $(76.74(76.56) \mathrm{kcal} / \mathrm{mol})$. The reactions in the aqueous phase are, in general, less endergonic than those in the gas phase (solvent effect). In the ionized form of cephalexin, demethylation (entry 3 ) and the loss of benzylamine carbamide (entry 5) are the most exergonic and appear to have almost the same spontaneity. The eliminations of benzylamine carbonyl (entry 7) and benzylamine carbamide (entry 5) and decarboxylation (entry 6) are very close in energy. According to Hammond's postulate [30], the structure of the transition state is similar for these three reactions. The negative values of Gibbs free energy $(\Delta G<0)$ mean that the pathway was exergonic processes (the release of energy from the reaction), indicating that the hydroxyl radical, $\bullet \mathrm{OH}$ adding to this reaction site P3 (Fig.9) may be more favored by thermodynamics, in addition, the Cephalexin molecule was ruptured (Entry 5) and then formed a series of stable products P4, P5 and P6. 


\subsection{Proposed mechanism of degradation}

Some authors have contributed to the study of the degradation byproducts of cephalexin, including theoretical studies, by using $\mathrm{FED}_{\mathrm{HOMO}}$ and $\mathrm{FED}_{\mathrm{LUMO}}$ to identify the reaction sites involved in radical attacks [2]. In this part, we are concerned with supporting our thermodynamic study by mechanistic research taking into account the abstraction, addition and CLX/CLX ${ }^{-}$fragmentation radicals previously indicated. Fig. 9 shows the mechanisms suggested in the generation of degradation byproducts (P1-P7).

The degradation byproduct P1 is formed by opening the cyclic amide after the addition of the $\mathrm{OH}$ radical to give the intermediate $\mathrm{R}_{\mathrm{add}}{ }^{\mathrm{c}}$, and the degradation reaction progresses to fragmentation (removal of $\mathrm{OH}$ ) or decarboxylation to form P3 from P2 [2,16,29]. The byproduct $\mathrm{P} 3$ reacts with the $\mathrm{OH}$ radical at $\mathrm{C} 14$ (hydroxylation reaction) passing to the $\mathrm{R}_{\text {add }}{ }^{\mathrm{d}}$ radical followed by removing $\mathrm{NH}_{2}$ (deamination) to generate $\mathrm{P} 7$. The other route is fragmentation (removal of benzylamine carbamide), which ends in dehydration to form P6. The theoretical results are well correlated with the previous experimental findings.

\subsection{Toxicity estimation}

The ecotoxicity of CLX and its byproducts to aquatic organisms was estimated at three trophic levels (fish, Daphnia and green algae) based on the European Union evaluation criteria for new chemical substances. The octanol-water partition coefficient $\left(\log K_{o w}\right)$ was predicted to be 0.4 for CLX, and this value is in good correlation with that reported in the literature obtained experimentally (0.65) [28,31].

The toxicities of degradation byproducts were classified according to the Globally Harmonized System of Classification and Labeling of Chemicals (GHS) [28], as shown in Table 9. The toxicity was measured for the degradation byproducts (P1-P7) as well as the starting molecule (cephalexin); the results are presented in Table 10.

The toxicity of degradation byproducts was examined for the three tropic levels (fish, daphnia and green algae). For green algae, 4 degradation byproducts (P1, P3, P4, and P5) are less toxic than CLX, and P2, P6, and P7 are more toxic. In the case of Daphnia, 5 degradation byproducts (P1, P2, P3, P4, and P5) are less toxic than CLX, and P6 and P7 are more toxic. For fish, 4 degradation byproducts (P1, P3, P4, and P5) are less harmful than CLX, and P2, P6, and P7 are more toxic.

As degradation continues, the toxicity worsens; furthermore, the degradation byproducts P1, P3, P4 and P5 are less toxic than cephalexin to all three organisms, while the byproducts P2, P6 and P7 are always more toxic than cephalexin, except for Daphnia, where P2 is less toxic. 
For green algae, all byproducts have an LC50/EC50 > $100 \mathrm{mg} / \mathrm{l}$ and are classified as nonharmful, with the exception of P7, which has an EC50 of 42.3 and is classified as harmful. CLX and most of the byproducts were found to be safe to aquatic organisms, and P7 is the only byproduct that is harmful to Daphnia. As a consequence, $\mathrm{ZnO}$ nanowire photocatalytic oxidation is a safe and clean process and is encouraged in the treatment of wastewater contaminated by cephalexin.

\section{Conclusion}

In this study, theoretical scale degradation of cephalexin was investigated in terms of reactivity, degradation thermodynamics, mechanism and ecotoxicological impact of degradation byproducts. It was revealed that the affinity of cephalexin toward addition reactions increases as it passes from the gas phase to aqueous phase and from the neutral to ionized form and decreases in the same way for the abstraction reactions. Thermodynamic data showed that the most favorable abstraction and addition radicals formed are those in which the reactant site is weakened by the inductive withdrawing effect, and the most stable radicals formed are those possessing larger mesomeric forms. The degradation byproducts (P1-P7) identified experimentally in the photocatalytic degradation of CLX by $\mathrm{ZnO}$ nanowires under simulated sunlight were verified in this computational work. The ecotoxicological impact of the degradation byproducts was clarified with ECOSAR software, where toxicity analysis revealed that CLX/CLX ${ }^{-}$was not toxic. Only its degradation product P7 was harmful, and the other oxidation byproducts (P1, P3, P4, and P5) were less toxic to fish and green algae. At the same time, P1, P2, P3, P4, and P5 were not toxic to Daphnia, and the rest of the byproducts were more toxic than cephalexin but still can be classified as nontoxic to the three organisms. Based on the collected information about the byproduct toxicities, we conclude that the photocatalytic degradation of cephalexin is a safe process for ecological organism studies. This study is the first to assess the safety of a photocatalytic process for the degradation of cephalexin based on a DFT approach toward the intermediate and byproduct structures of the degradation process. 


\section{Statements \& Declarations}

\section{Funding}

We would like to thank the financial support of Direction Générale de la Recherche Scientifique et du Développement Technologique (DGRSDT) of Algeria through the national research programs (PRFU N ${ }^{\circ}$ B00L01UN180120220001).

\section{Competing Interests}

The authors have no relevant financial or non-financial interests to disclose

\section{Author Contributions}

R. Masmoudi: Investigation, Methodology, Writing- Original draft. Writing - reviewing and editing. S. Khettaf: Methodology, Validation. A. Soltani: Formal analysis, Validation. A. Dibi: Conceptualization, Validation. L. Messaadia: Software, Formal analysis. M. Benamira: Supervision, Validation, Writing - reviewing and editing.

\section{Data Availability}

The datasets generated during and/or analysed during the current study are available from the corresponding author on reasonable request.

\section{Code availability}

Gaussian 16 program package: $\mathrm{N}^{\circ}$ Licence G32214419065828W-4627N, G16W Full Version, 32-Bit, Rev: A.03 Front: 1.1. Version: Multiprocessor. GaussView6 for Windows, Rev: 6.0.16, 32-bit. V32108462166828W-4627N. Ecosar Class Program (Free Ware).

\section{References}

1. Bailey, A. Walker, A. Hadley, D.G. James, Cephalexin. A new oral antibiotic., Torax. 20 (1971) 15-19.

2. J. He, Y. Zhang, Y. Guo, G. Rhodes, J. Yeom, H. Li, W. Zhang, Photocatalytic degradation of cephalexin by $\mathrm{ZnO}$ nanowires under simulated sunlight: Kinetics, influencing factors, and mechanisms, Environ. Int. 132 (2019) 105105. doi: 10.1016/j.envint.2019.105105. 
3. A.L. Estrada, Y.-Y. Li, A. Wang, Biodegradability enhancement of wastewater containing cefalexin by means of the electro-Fenton oxidation process, J. Hazard. Mater. 227 (2012) 41-48. doi: 10.1016/j.jhazmat.2012.04.079.

4. N. Benarab, F.F. Fangninou, The issues of antibiotics: cephalexin antibiotic as emerging environment contaminant, Int. J. Sci. Res. Publ. IJSRP. 10 (2020).

5. K. Ikehata, N. Jodeiri Naghashkar, M. Gamal El-Din, Degradation of aqueous pharmaceuticals by ozonation and advanced oxidation processes: a review, Ozone Sci. Eng. 28 (2006) 353-414. doi: 10.1080/01919510600985937.

6. T. Mandal, S. Maity, D. Dasgupta, S. Datta, Advanced oxidation process and biotreatment: Their roles in combined industrial wastewater treatment, Desalination. 250 (2010) 87-94. doi: 10.1016/j.desal.2009.04.012.

7. M.I. Stefan, Advanced oxidation processes for water treatment: fundamentals and applications, IWA publishing, 2017. doi: 10.2166/9781780407197_0297.

8. D. Kanakaraju, B.D. Glass, M. Oelgemöller, Advanced oxidation process-mediated removal of pharmaceuticals from water: A review, J. Environ. Manage. 219 (2018) 189-207. https://doi.org/10.1016/j.jenvman.2018.04.103. doi: 10.1016/j.jenvman.2018.04.103.

9. J. Cao, Q. Ren, F. Chen, T. Lu, Comparative study on the methods for predicting the reactive site of nucleophilic reaction, Sci. China Chem. 58 (2015) 1845-1852. doi: 10.1007/s11426015-5494-7.

10. W. Yang, W.J. Mortier, The use of global and local molecular parameters for the analysis of the gas-phase basicity of amines, J. Am. Chem. Soc. 108 (1986) 5708-5711.

11. C. Droguett, R. Salazar, E. Brillas, I. Sirés, C. Carlesi, J.F. Marco, A. Thiam, Treatment of antibiotic cephalexin by heterogeneous electrochemical Fenton-based processes using chalcopyrite as sustainable catalyst, Sci. Total Environ. 740 (2020) 140154. https://doi.org/https://doi.org/10.1016/j.scitotenv.2020.140154.

12. P. Bansal, A. Verma, N, Ag co-doped TiO2 mediated modified in-situ dual process (modified photocatalysis and photo-Fenton) in fixed-mode for the degradation of Cephalexin under solar irradiations, Chemosphere. 212 (2018) 611-619. https://doi.org/https://doi.org/10.1016/j.chemosphere.2018.08.120.

13. H. Wu, Q. Feng, H. Yang, E. Alam, B. Gao, D. Gu, Modified biochar supported Ag/Fe nanoparticles used for removal of cephalexin in solution: Characterization, kinetics and mechanisms, Colloids Surfaces A Physicochem. Eng. Asp. 517 (2017) 63-71. https://doi.org/https://doi.org/10.1016/j.colsurfa.2017.01.005.

14. L.A. Perea, R.E. Palma-Goyes, J. Vazquez-Arenas, I. Romero-Ibarra, C. Ostos, R.A. Torres-Palma, Efficient cephalexin degradation using active chlorine produced on ruthenium and iridium oxide anodes: Role of bath composition, analysis of degradation pathways and degradation extent, Sci. Total Environ. 648 (2019) 377-387. https://doi.org/https://doi.org/10.1016/j.scitotenv.2018.08.148.

15. D.A.C. Coledam, M.M.S. Pupo, B.F. Silva, A.J. Silva, K.I.B. Eguiluz, G.R. Salazar-Banda, J.M. Aquino, Electrochemical mineralization of cephalexin using a conductive diamond anode: a mechanistic and toxicity investigation, Chemosphere. 168 (2017) 638-647.

16. V.S. Antonin, J.M. Aquino, B.F. Silva, A.J. Silva, R.C. Rocha-Filho, Comparative study on the degradation of cephalexin by four electrochemical advanced oxidation processes: 
evolution of oxidation intermediates and antimicrobial activity, Chem. Eng. J. 372 (2019) 1104-1112. doi: 10.1016/j.cej.2019.04.185.

17. W. Guo, H. Wang, Y. Shi, G. Zhang, Sonochemical degradation of the antibiotic cephalexin in aqueous solution, Water Sa. 36 (2010). doi: 10.4314/wsav36i5.61998.

18. M.G. Alalm, A. Tawfik, S. Ookawara, Degradation of four pharmaceuticals by solar photo-Fenton process: kinetics and costs estimation, J. Environ. Chem. Eng. 3 (2015) 4651.

19. A.D. Becke, Density-functional thermochemistry. IV. A new dynamical correlation functional and implications for exact-exchange mixing, J. Chem. Phys. 104 (1996) 10401046. https://doi.org/10.1063/1.470829. doi: 10.1063/1.470829.

20. J. Ho, M.Z. Ertem, Calculating free energy changes in continuum solvation models, J. Phys. Chem. B. 120 (2016) 1319-1329. doi: 10.1021/acs.jpcb.6b00164.

21. T. Yamana, A. Tsuji, Comparative stability of cephalosporins in aqueous solution: Kinetics and mechanisms of degradation, J. Pharm. Sci. 65 (1976) 1563-1574. https://doi.org/10.1002/jps.2600651104.

22. R.K. Roy, S. Pal, K. Hirao, On non-negativity of Fukui function indices, J. Chem. Phys. 110 (1999) 8236-8245. doi: 10.1063/1.478792.

23. A. Galano, J.R. Alvarez-Idaboy, Guanosine $+\mathrm{OH}$ radical reaction in aqueous solution: A reinterpretation of the UV-vis data based on thermodynamic and kinetic calculations, Org. Lett. 11 (2009) 5114-5117. doi: 10.1021/o1901862h.

24. T. An, Y. Gao, G. Li, P. V Kamat, J. Peller, M. V Joyce, Kinetics and mechanism of $\bullet \mathrm{OH}$ mediated degradation of dimethyl phthalate in aqueous solution: experimental and theoretical studies, Environ. Sci. Technol. 48 (2014) 641-648.

25. H. Sanderson, D.J. Johnson, C.J. Wilson, R.A. Brain, K.R. Solomon, Probabilistic hazard assessment of environmentally occurring pharmaceuticals toxicity to fish, daphnids and algae by ECOSAR screening, Toxicol. Lett. 144 (2003) 383-395. doi: 10.1016/S03784274(03)00257-1.

26. T.J. Al-Musawi, H. Kamani, E. Bazrafshan, A.H. Panahi, M.F. Silva, G. Abi, Optimization the effects of physicochemical parameters on the degradation of cephalexin in sono-Fenton reactor by using box-Behnken response surface methodology, Catal. Letters. 149 (2019) 1186-1196.

27. M.S. Legnoverde, S. Simonetti, E.I. Basaldella, Influence of $\mathrm{pH}$ on cephalexin adsorption onto SBA-15 mesoporous silica: Theoretical and experimental study, Appl. Surf. Sci. 300 (2014) 37-42.

28. S.A. Cinar, A. Ziylan-Yavaş, S. Catak, N.H. Ince, V. Aviyente, Hydroxyl radical-mediated degradation of diclofenac revisited: a computational approach to assessment of reaction mechanisms and by-products, Environ. Sci. Pollut. Res. 24 (2017) 18458-18469. doi: 10.1007/s11356-017-9482-7.

29. F. Tavasol, T. Tabatabaie, B. Ramavandi, F. Amiri, Design a new photocatalyst of sea sediment/titanate to remove cephalexin antibiotic from aqueous media in the presence of sonication/ultraviolet/hydrogen peroxide: Pathway and mechanism for degradation, Ultrason. Sonochem. 65 (2020) 105062. doi: 10.1016/j.ultsonch.2020.105062.

30. D. Farcasiu, The use and misuse of the Hammond postulate, J. Chem. Educ. 52 (1975) 76. doi: 10.1021/ed052p76. 
31. K. Kümmerer, Pharmaceuticals in the environment: sources, fate, effects and risks, Springer Science \& Business Media, 2008. 
Figures

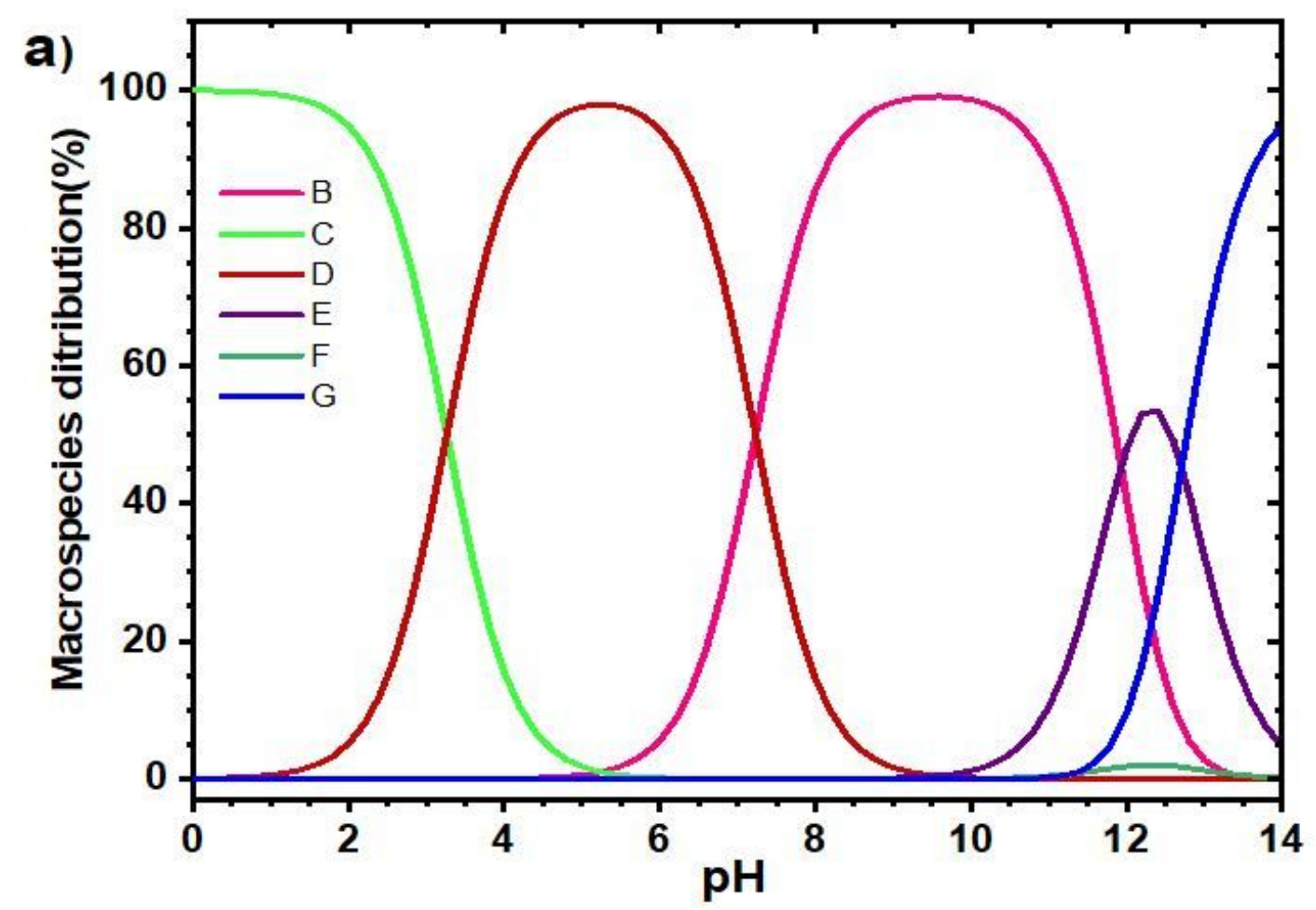

b)

B<smiles>CC1=C(C(=O)[O-])N2C(=O)[C@H](CC(=O)[C@H](C)c3ccccc3)[C@H]2CC1</smiles>

$99.09 \%$

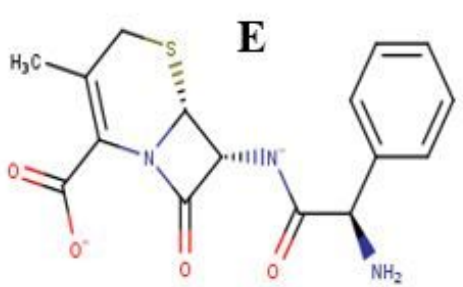

$53.43 \%$
C

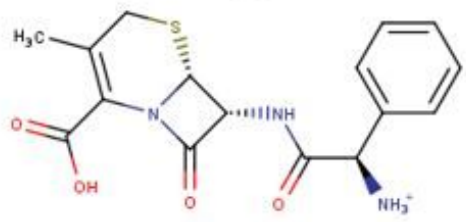

$99.91 \%$

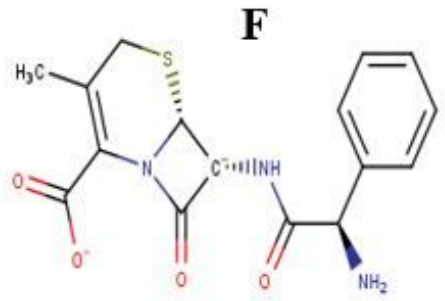

$12.40 \%$
D

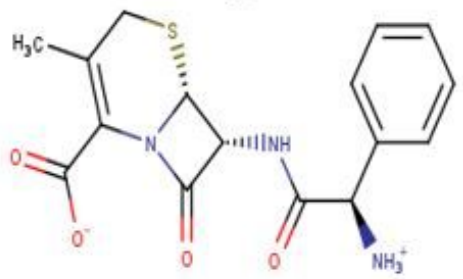

$97.95 \%$

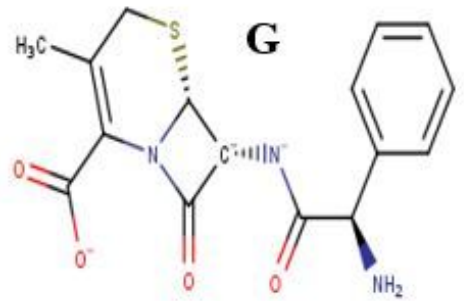

$94.97 \%$

Figure 1

a) Species distribution diagram of cephalexin as a function of solution $\mathrm{pH}$. b) Molar fractions (\%) of the main different species ofcephalexin. 


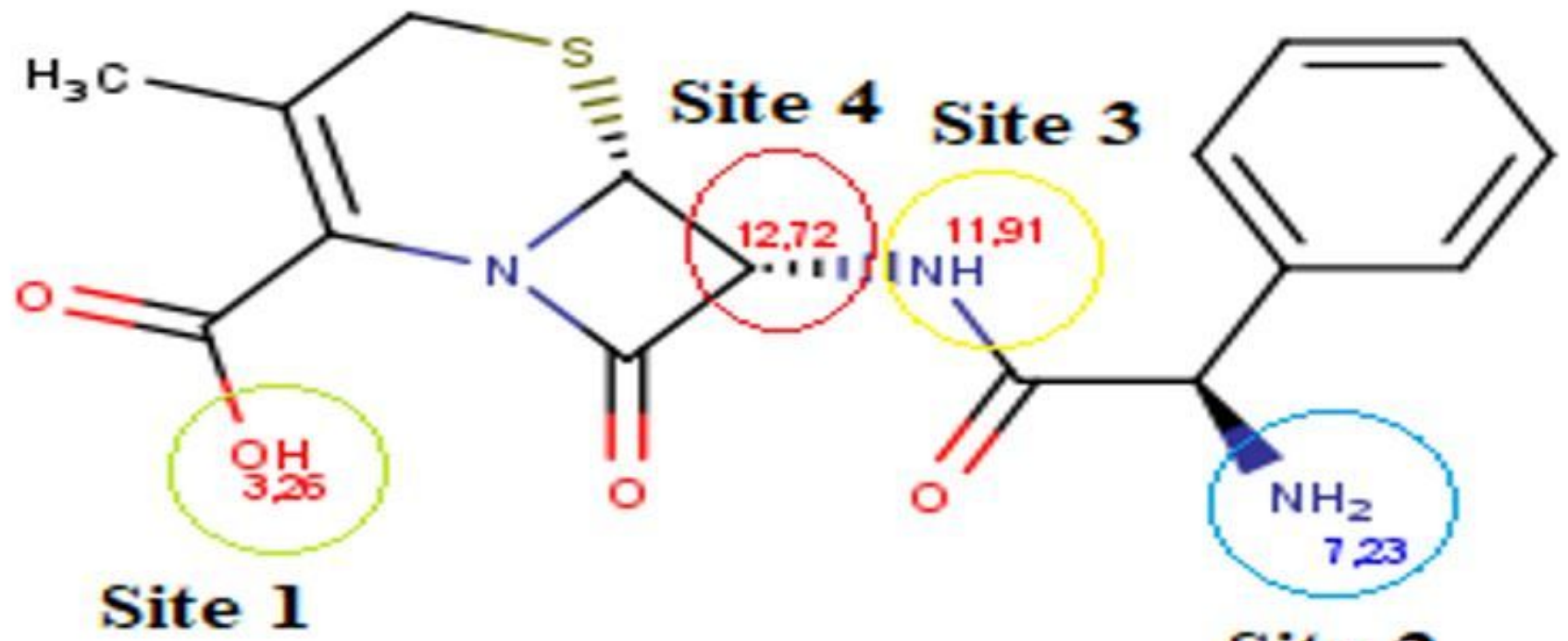

Site 2

Figure 2

The 2D structure of CLX
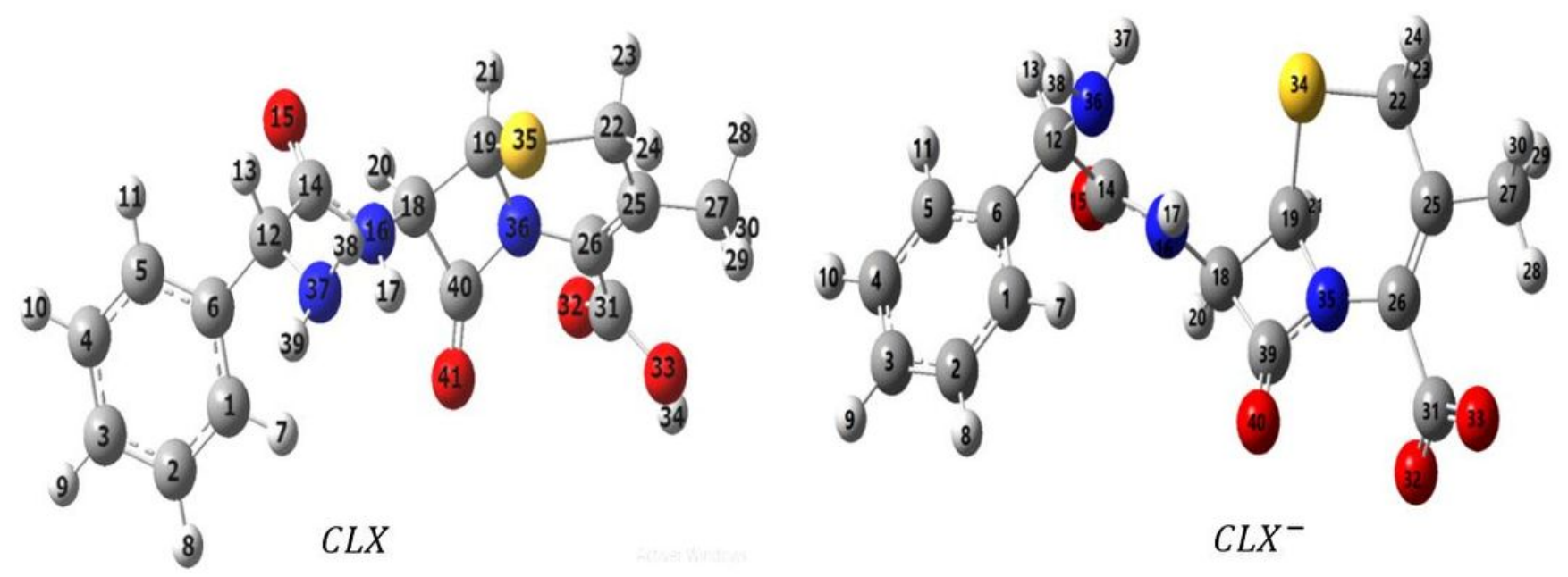

Figure 3

The optimized structure of CLX/CLX using DFT/B3LYP/6-31+G(d) 
(1)

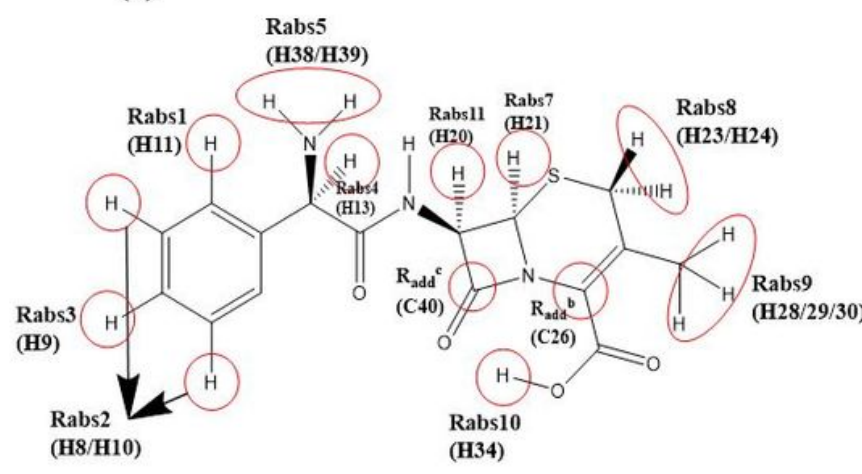

(2)

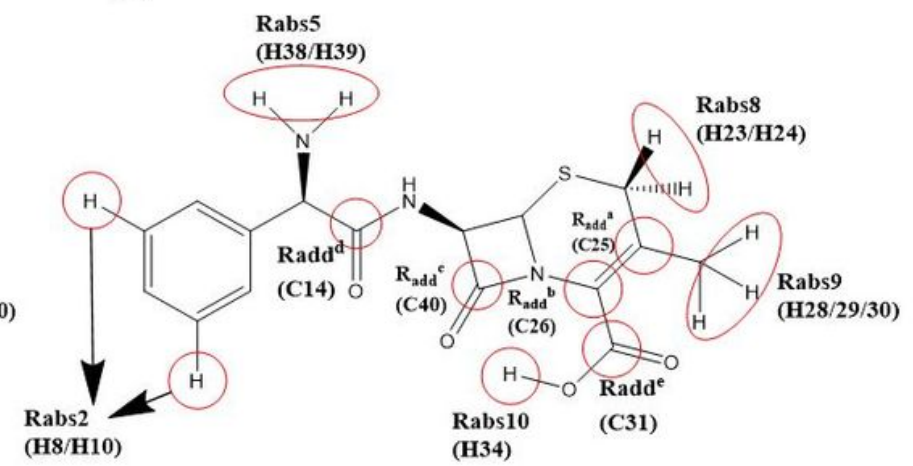

Figure 4

The sites concerned with radical attack on neutral cephalexin in gas (1) and aqueous phase (2)

(3)

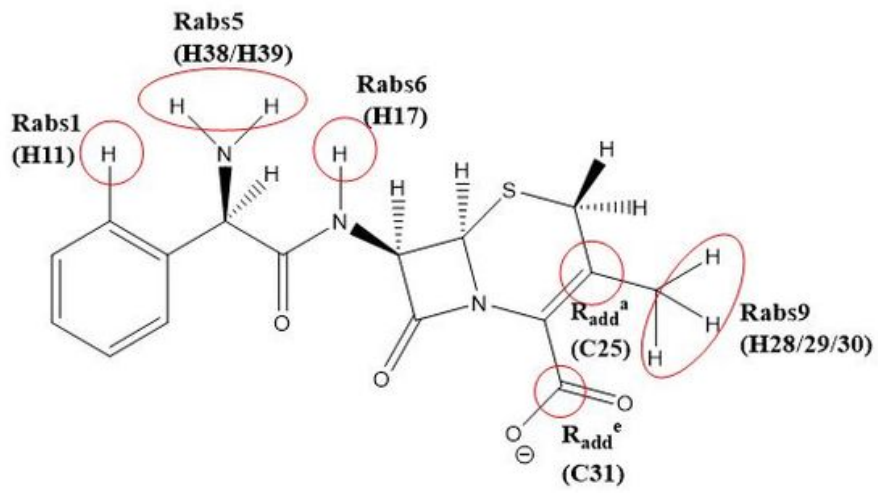

(4)

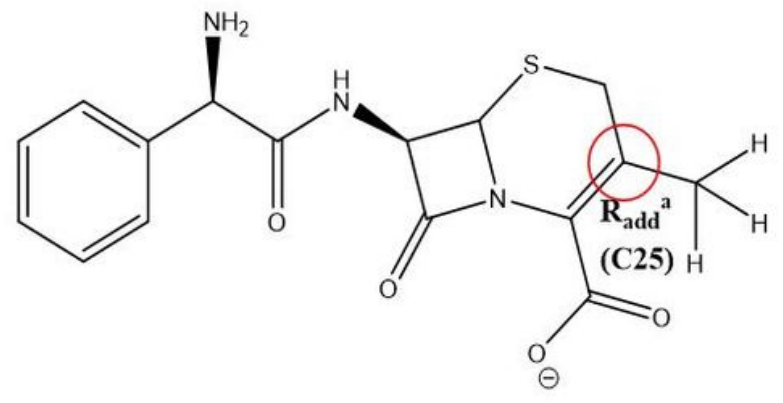

\section{Figure 5}

The sites concerned with radical attack on ionized cephalexin in gas (3) and aqueous phase (4)

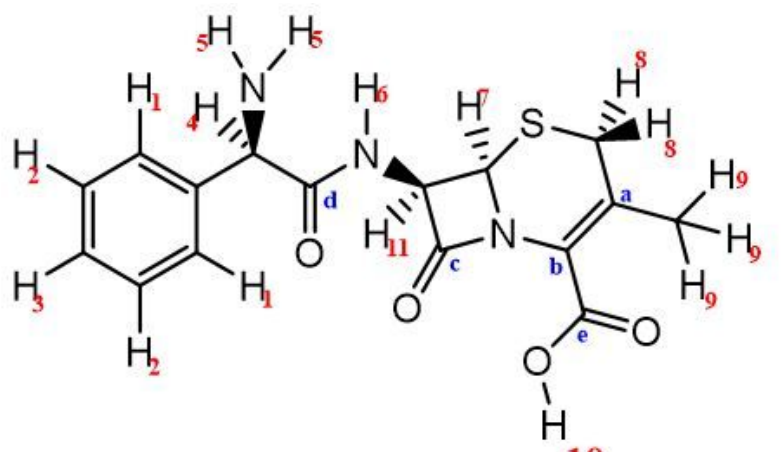

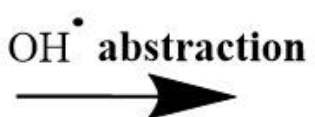<smiles></smiles> 
Figure 6

$\mathrm{H}$-abstraction by $\cdot \mathrm{OH}$ from $\mathrm{CLX}$, generating radical intermediate $\left(R_{\mathrm{abs}}\right)$

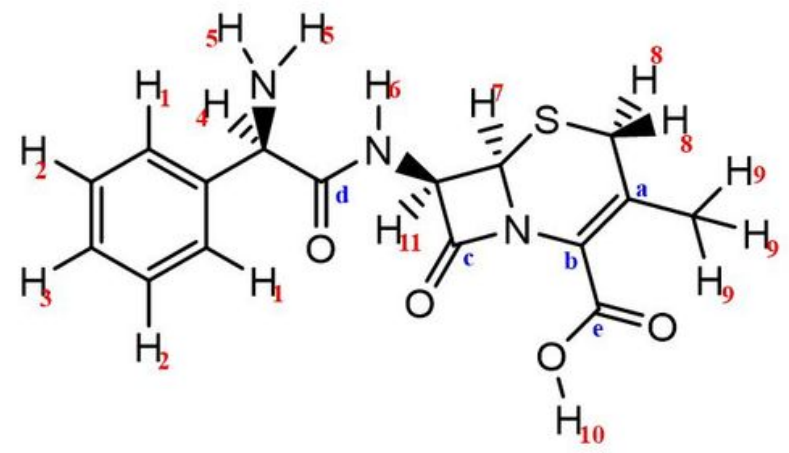

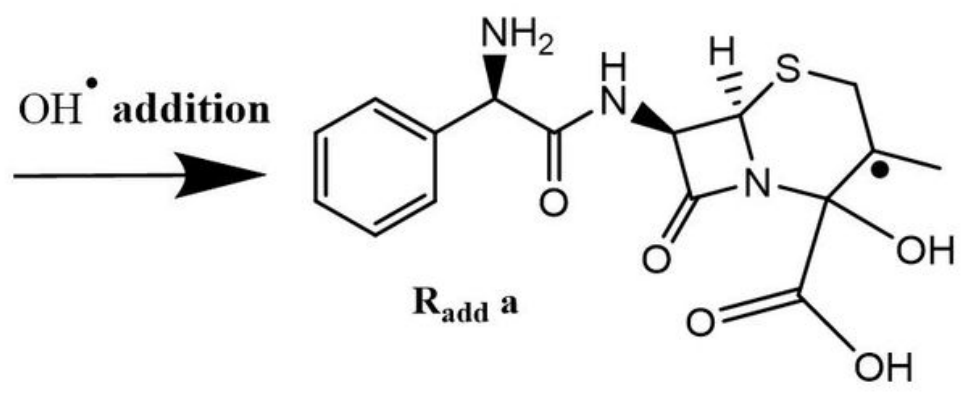

Figure 7

$\mathrm{OH}$ addition by $\cdot \mathrm{OH}$ from CLX, generating radical intermediate $\left(R_{\text {add }}\right)$

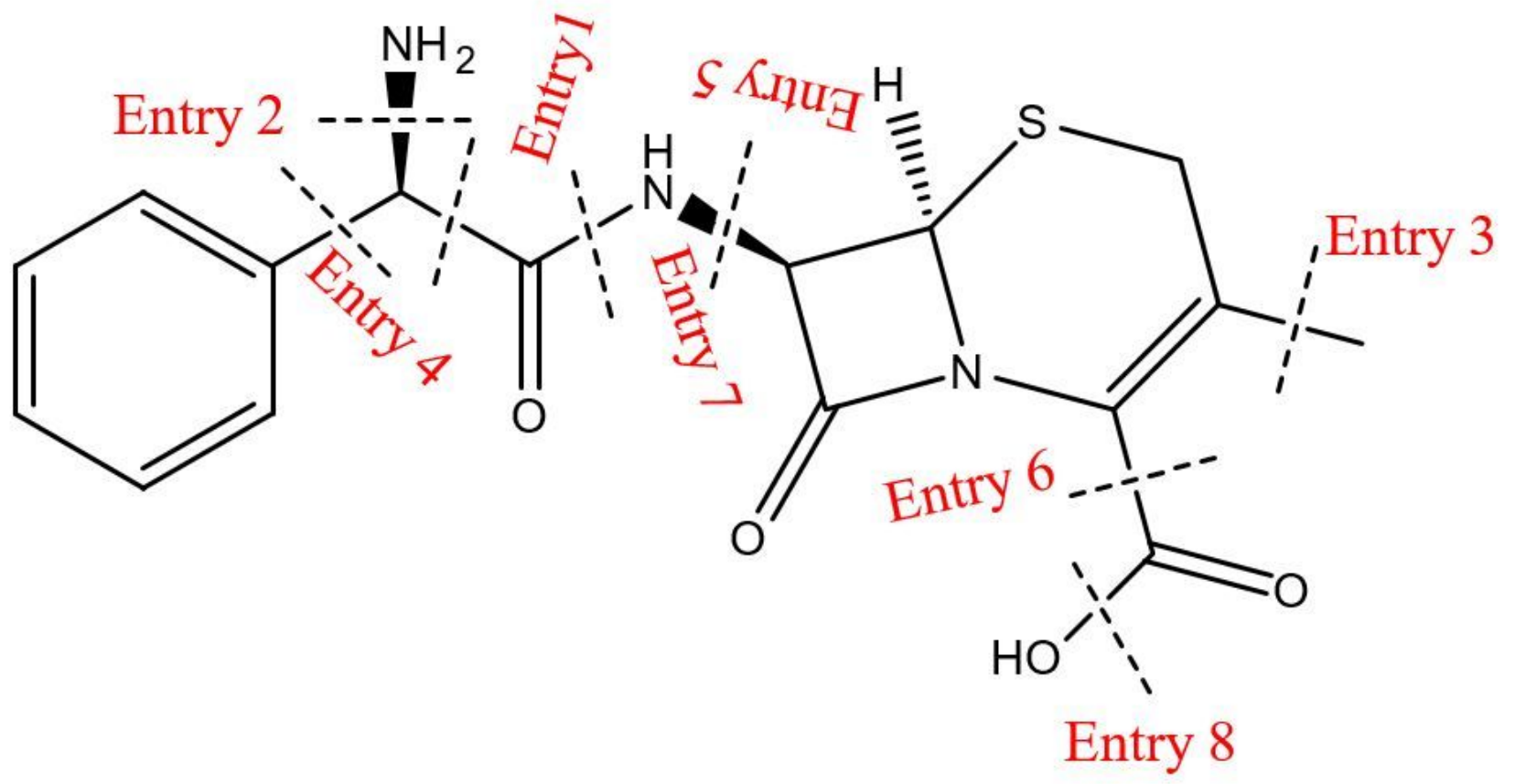

Figure 8

Fragmentation pathways of CLX/CLX 


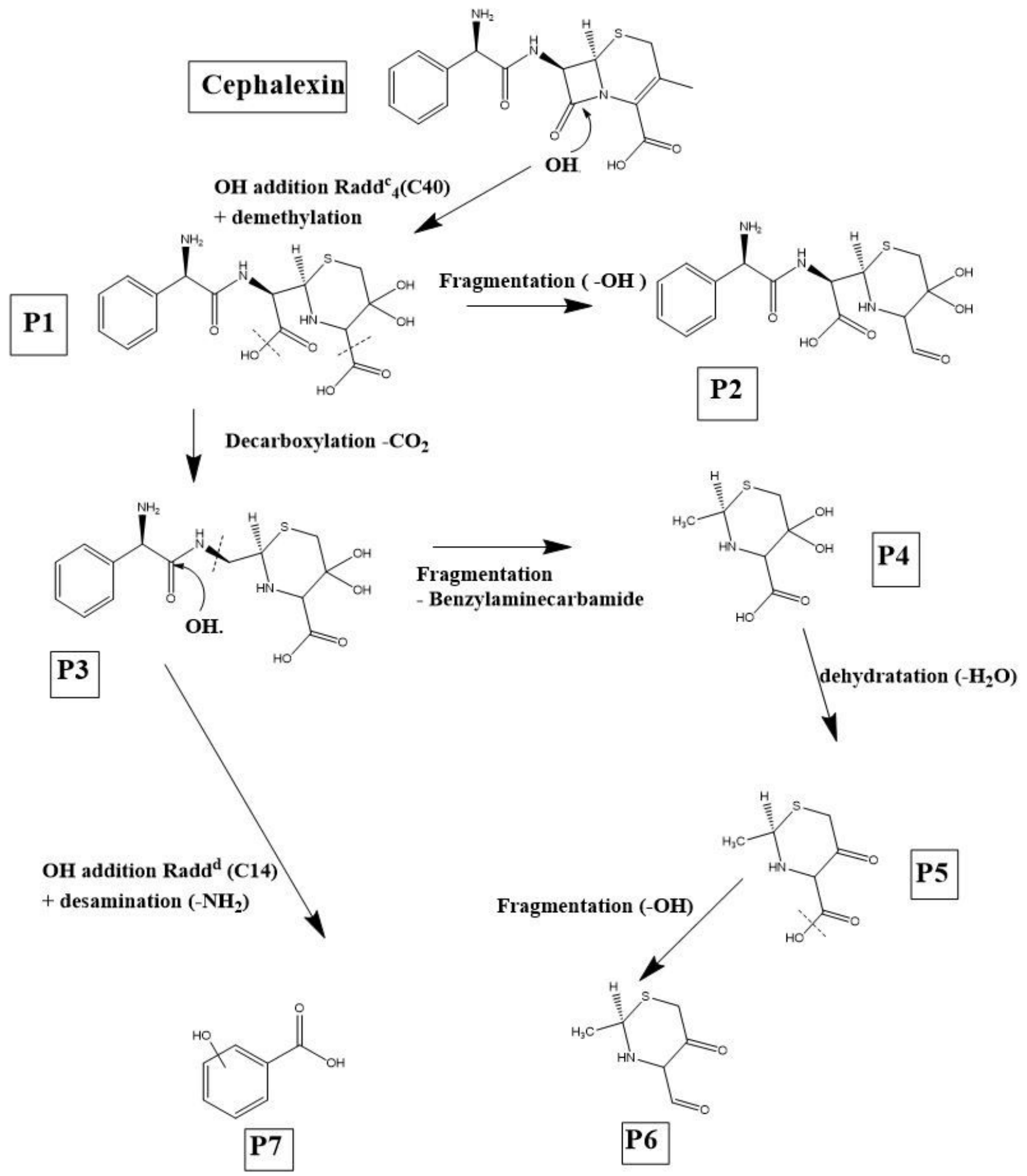

Figure 9

Proposed mechanism of cephalexin degradation. 
This is a list of supplementary files associated with this preprint. Click to download.

- GraphicalAbstract.docx 